\title{
Un nouvel esprit pour le capitalisme : la société de l'information?
}

\section{Olivier Simioni}

\section{(2) OpenEdition}

\section{Journals}

Édition électronique

URL : http://journals.openedition.org/ress/616

DOI : $10.4000 /$ ress.616

ISSN : 1663-4446

Éditeur

Librairie Droz

Édition imprimée

Date de publication : 1 juin 2002

Pagination : 75-90

ISBN : 2-600-00693-1

ISSN : 0048-8046

\section{Référence électronique}

Olivier Simioni, «Un nouvel esprit pour le capitalisme : la société de l'information? 》, Revue européenne des sciences sociales [En ligne], XL-123 | 2002, mis en ligne le 03 décembre 2009, consulté le 30 avril 2019. URL : http://journals.openedition.org/ress/616 ; DOI : 10.4000/ress.616 


\section{Olivier SIMIONI}

\section{UN NOUVEL ESPRIT POUR LE CAPITALISME: LA SOCIÉTÉ DE L'INFORMATION?}

Depuis que l'expression «société de l'information» est apparue, probablement au Japon au début des années 1960 (voir Duff 2000), on ne compte plus le nombre d'ouvrages, articles ou rapports qui ont tenté de cerner les contours de ce phénomène difficile à saisir. Une première approche de cette multitude de discours sur le «nouvel âge de l'information» donne l'impression d'une grande hétérogénéité dont les seuls liants seraient la constatation d'une convergence de l'informatique et des télécommunications, d'un développement de ces «nouvelles» technologies de l'information et de la communication (NTIC) ainsi qu'une référence continuelle au changement (voir Simioni 2000). Nous serions en train de vivre une période de rupture, mutation, multiples transformations fondamentales, révolution, le tout étant déterminé, en grande partie, par le développement de plus en plus rapide des technologies. L'importance de ces changements serait telle que l'on pourrait carrément diviser l'histoire de l'humanité en trois parties: ère agricole, ère industrielle et ère de l'information. Les enjeux théoriques et pratiques sont donc de taille et il n'est pas étonnant que tant de futurologues, gouvernements et autres producteurs de discours aient voulu apporter leur pierre à l'édifice.

Si l'on étudie tous ces documents d'un peu plus près, on se rend compte que, derrière des expressions ${ }^{1}$ ou atours souvent différents, indépendamment des nouvelles technologies et de la constatation d'une rupture, se cache peut-être malgré tout une vision du monde commune. On peut constater la présence d'un modèle récurrent de société et d'individu qui se matérialise, par exemple, dans des expressions vagues comme décentralisation, démassification, réseau, flexibilité ou changement permanent. Ce modèle nous rappelle étrangement celui proposé par Luc Boltanski et Ève Chiapello dans «Le nouvel esprit du capitalisme» (1999) pour qualifier la «cité par projets» qu'ils ont extraite de la littérature de management des années 1990. Or, cette «cité par projets» ne serait qu'un nouveau support de l'esprit du capitalisme dont on pourrait repérer au moins deux autres formes aux $19^{\mathrm{e}}$ et $20^{\mathrm{e}}$ siècles. L'aspect a priori révolutionnaire des thèmes traités par les discours sur la société de l'information est remis à un niveau peut-être plus judicieux ou pertinent. Il y a certes transformation mais on ne peut pas pour autant parler de révolution ou, pire, de changement de civilisation.

La société de l'information telle qu'on nous la présente trop souvent n'est pas un bon point de départ pour décrire et étudier la société d'aujourd'hui. Cette

1 On parle aussi bien de société de l'information que de société en réseau, société du savoir, cybermonde, société digitale, etc. 
approche pose de trop grands problèmes d'analyse. Mais il faut aller plus loin. En effet, les discours qui la défendent sont loin d'être d'innocentes fables. Ils apparaissent plutôt comme une forme de discours d'accompagnement encourageant et justifiant la réalisation de certains idéaux politiques. Or, ces idéaux politiques et leurs conséquences ne correspondent pas forcément à ce que peut imaginer de prime abord le lecteur ou auditeur visé. On pourrait même dire que l'on a affairelà à des discours idéologiques, en ce sens qu'il y aurait une forme de distorsion (voire de travestissement) de la réalité dans un but de légitimation. Cette hypothèse est peut-être excessive et elle a certainement pour défaut de réduire la complexité de la problématique. Néanmoins, elle peut être prise en considération dans un certain nombre de cas. Ceci dit, un détour par l'ouvrage de Luc Boltanski et Ève Chiapello s'avère utile.

\section{UN NOUVEL ESPRIT POUR LE CAPITALISME}

«Le nouvel esprit du capitalisme» est un ouvrage extrêmement touffu et complexe et il serait vain de vouloir le résumer en quelques lignes. En fait, les éléments qui nous intéressent plus particulièrement sont, premièrement, de savoir ce qu'est un «esprit» du capitalisme et, deuxièmement, de dégager les traits essentiels de la «cité par projets» ou, plus largement, du «nouvel esprit du capitalisme».

Pour les deux auteurs qui s'inspirent de Max Weber, la marque première du capitalisme est l'«exigence d'accumulation illimitée du capital par des moyens formellement pacifiques. C'est la remise en jeu perpétuelle du capital dans le circuit économique dans le but d'en tirer un profit, c'est-à-dire d'accroître le capital qui sera à son tour réinvesti » (1999: 37). Ce système a quelque chose d'absurde car il demande la mobilisation d'un très grand nombre de personnes dont les chances de profit peuvent paraître faibles. Pour les deux auteurs, l'existence d'un esprit du capitalisme est nécessaire pour pouvoir motiver les travailleurs « compte tenu du caractère singulier, voire transgressif, des modes de comportement exigés par le capitalisme par rapport aux formes de vie attestées dans la plupart des sociétés humaines»(1999: 43). Ils ne considèrent pas que le seul salaire ou la contrainte puissent être des motifs suffisants. Il semble nécessaire d'en référer à de puissantes raisons morales pour que les personnes se rallient au capitalisme, d'en référer à un esprit défini comme «l'idéologie qui justifie l'engagement dans le capitalisme » (1999: 42). Idéologie est compris ici non pas dans «le sens réducteur - auquel l'a souvent ramené la vulgate marxiste - d'un discours moralisateur visant à voiler des intérêts matériels et sans cesse démenti par les pratiques » mais dans celui «d'un ensemble de croyances partagées, inscrites dans des institutions, engagées dans des actions et par là ancrées dans le réel» (1999: 35). Cette distinction a son intérêt pour la suite.

En attendant, on peut tenter de dégager les traits principaux des esprits du capitalisme qui se sont succédés. Pour Boltanski et Chiapello, il y a une base stable, des piliers; ce sont les idées de progrès (économique, technique, scientifique), d'efficacité économique d'une production stimulée par la concurrence ainsi que les libertés individuelles. Mais cette base, trop générale, n'est pas suffisante pour engager les personnes ordinaires dans leurs activités quotidiennes, en particulier 
au travail. Les personnes ont besoin d'arguments plus forts, de justifications, de réponses, notamment aux trois questions suivantes:

- En quoi l'engagement dans les processus d'accumulation capitaliste est-il source d'enthousiasme, y compris pour ceux qui ne seront pas nécessairement les premiers bénéficiaires des profits réalisés?

- Dans quelle mesure ceux qui s'impliquent dans le cosmos capitaliste peuventils être assurés d'une sécurité minimale pour eux et leurs enfants?

- Comment justifier, en termes de bien commun, la participation à l'entreprise capitaliste et défendre, face aux accusations d'injustice, la façon dont elle est animée et gérée? (1999: 53-54).

On repère là des éléments d'engagement, de sécurité et de justice. Ce dernier élément est au centre de l'ouvrage. En effet, «les agencements sociétaux, dans la mesure où ils sont soumis à un impératif de justification, tendent à incorporer la référence à un type de conventions très générales orientées vers un bien commun et prétendant à une validité universelle, modélisée sous le concept de cité. Le capitalisme ne fait pas exception » (1999: 61).

Les « cités » font partie d'un modèle théorique complexe que l'on ne peut présenter ici. Ce qu'il faut relever, c'est l'existence de plusieurs «cités» dont les noms sont parfois évocateurs: cités inspirée, marchande, du renom, domestique, industrielle ou civique (voir Boltanski et Thévenot 1991). Les différentes formes que prend l'esprit du capitalisme dans l'histoire s'inspirent de ou reposent en partie sur ces modèles. Or, en étudiant la littérature de management des années 1990, destinée aux cadres désignés comme porte-parole du capitalisme, Boltanski et Chiapello ont estimé nécessaire de dégager ce qui apparaît comme un nouveau modèle idéal-typique du bien commun: la «cité par projets».

Avant d'en venir à la présentation des particularités de cette nouvelle « cité » et de l'esprit qui la contient, on peut rajouter que les deux auteurs, en plus des descriptions de Max Weber (1964), ont repéré deux autres descriptions d'un esprit du capitalisme. La première se rapporterait plus particulièrement à la fin du $19^{\mathrm{e}}$ siècle et la seconde aux années 1930-1960. Si décrire la première ne nous serait pas d'une grande utilité ici, il est intéressant de relever quelques traits saillants de la seconde. En effet, on peut admettre avec les auteurs que l'esprit du capitalisme se nourrit de sa propre critique et que, ainsi, le nouvel esprit est, en partie, une tentative de réponse aux critiques adressées à son prédécesseur. Ce précédent esprit peut être assez rapidement imaginé en mettant l'accent sur certains termes: organisation, centralisation, bureaucratie, planification, directeur (salarié et non pas détenteur du capital), cadres, ingénieurs, hiérarchie, production de masse, consommation de masse, standardisation. Les éléments d'engagement sont la possibilité d'accéder à des positions de pouvoir importantes ou la réalisation des désirs grâce à la production et à la consommation de masse. Du point de vue de la sécurité, c'est en particulier la perspective de carrière ainsi que la prise en charge de la vie quotidienne dans le cadre d'organisations de plus en plus gigantesques qui dominent. Enfin, du point de vue du bien commun, on note l'importance de la solidarité institutionnelle qui permet de penser à l'atténuation ou même à la fin de la lutte des classes. 
Le nouvel esprit du capitalisme se construit donc, en partie, en opposition à tout cela. Les textes de management des années 1990 cherchent à motiver tous les employés et non plus seulement les cadres. Ils critiquent la bureaucratie, rejettent les hiérarchies (pyramidales) et la planification, insistent sur la concurrence, le changement permanent, défendent l'organisation flexible, l'idée d'adaptabilité. L'entreprise type est maigre, elle travaille en réseau, sans unité de lieu ni de temps, et s'organise par équipes ou par projets. On travaille moins au bureau, les équipes s'auto-organisent, s'auto-contrôlent, les figures du patron ou du cadre traditionnels perdant totalement de leur valeur. Ce sont les leaders, coachs ou managers, capables de se mouvoir dans l'incertitude ou la complexité, qui coordonnent, qui créent de l'enthousiasme. On ne parle donc plus de stabilité ou de structure mais plutôt d'intuition créatrice, d'innovation, de mobilité. Les travailleurs doivent s'auto-motiver, se responsabiliser, se mobiliser; ils se forment en permanence, ce sont des caméléons. Une des compétences essentielles est de savoir où et quelles informations chercher. En effet, l'information devient une source de productivité et de profit de premier plan.

La «cité par projets», comme modèle de justification, permet de répondre en partie aux critiques qui pourraient être adressées à ce nouvel esprit. En passant d'un projet à l'autre, les individus acquièrent de l'employabilité car ils augmentent leur réseau, leur capacité à créer des liens dans un monde connexionniste. À un autre niveau, la standardisation et les formes d'inauthenticité ou de désenchantement qui sont liées à la production et à la consommation de masse sont supprimées par une production flexibilisée, tenant compte des désirs individuels. Les «petits chefs » autoritaires disparaissent et les qualités personnelles, et non plus seulement les compétences spécifiques, sont mieux prises en compte dans le travail $^{2}$. On imagine donc certaines formes de libération...

On reviendra sur les problèmes engendrés par cette nouvelle vision du monde. Ce que l'on peut déjà dire, c'est que, au niveau des discours, il y a une rupture évidente que l'on pourrait situer dans les années 1970-1980, notamment après les événements de mai 1968. Or, première indication, c'est à peu près à ce moment-là que l'on peut situer l'apparition des textes «fondateurs » de la société de l'information.

\section{«SOCIÉTÉ DE L'INFORMATION»}

Si on peut remonter très loin pour découvrir des indices des origines des discours concernant la société de l'information, on doit toutefois admettre que l'apparition de cette expression et des thèmes liés se fait en particulier dans les années 1960. En effet, bien que le thème du réseau soit déjà développé au $19^{\mathrm{e}}$ siècle, que la cybernétique de Norbert Wiener ou les théories de Claude Shannon ou Alan Turing montrent le rôle prépondérant de l'information ou de la communication dès les années 1940 (voir, par exemple, Mattelart 2001 ou Musso 1999), le thème d'une société de l'information en tant que tel apparaît en premier lieu dans certains discours au Japon et aux Etats-Unis à la fin des années 1960 et au début des

2 Ce qui veut aussi dire, corrélativement, que la séparation entre la vie privée et la vie professionnelle aurait tendance à s'atténuer. 
années 1970 (voir Simioni 2000 et Duff 2000). A partir de là, on va assister à un véritable éclatement des discours sur la société de l'information. On ne compte plus, aujourd'hui, le nombre de livres, articles, rapports ou autres documents qui traitent de la question. Il devient d'autant plus difficile de trouver une ligne directrice qui rendrait compte des spécificités de «la» société de l'information. En fait, en première lecture, on pourrait être tenté de n'y voir que multiples variations autour des NTIC et de l'idée qu'une transformation importante est en cours. Mais que peut-on en tirer de plus?

Il peut sembler judicieux de commencer par chercher un lien du côté de la question des rapports entre Etat et Marché. En effet, le thème de la «global marketplace », de la libéralisation ou de la déréglementation est largement présent dans les discours sur la société de l'information. On imagine que la doctrine néolibérale a fait son chemin et s'impose notamment par cette voie. Mais on se rend vite compte que ce n'est pas une approche suffisante bien qu'elle soit nécessaire, on le verra plus tard. Les textes estimant que le rôle de l'Etat est important ne sont pas rares et l'accent est plutôt mis sur le subtil mélange entre compétition et coopération qui s'est notamment matérialisé dans ce néologisme qu'est la «coopétition». De plus, la logique marchande est «concurrencée» sur son terrain anti-étatiste par des discours à forte connotation libertaire. En fait, une analyse plus poussée nous incite à mettre l'accent sur d'autres éléments structurants. On peut en effet repérer la quasi omniprésence de ce qu'on pourrait appeler une nébuleuse sémantique ${ }^{3}$ dont l'expression phare serait « décentralisation». On peut tenter de décrire rapidement l'idée générale dont on trouve des traces dans une grande majorité de textes consacrés à la société de l'information. Le constat est celui d'une lourdeur bureaucratique et d'une hiérarchisation, qui ne sont plus adaptées à la vitesse des transformations actuelles et aux aspirations des individus. Les termes de réseaux, d'horizontalité ou même de constellation, indiquent le refus des logiques pyramidales. Les nouvelles technologies devraient permettre l'émergence d'une démocratie participative basée, par exemple, sur le vote à domicile et le référendum continu. Le niveau local ou communautaire de décision devrait être privilégié, renforcé. Pour beaucoup, on verra aussi l'émergence d'un nouvel individu, responsable, créatif et ayant le sens de l'initiative. Finis la planification rigide et le temps des masses; nous rentrons dans une époque de projets, d'adaptabilité, de mobilité, de fluidité et de flexibilité. On assistera à un développement de l'esprit d'entreprise et les nouvelles technologies devraient donc permettre l'accomplissement individuel et le déploiement des facultés personnelles. Un autre point important consiste à dire que l'apprentissage ou la formation ne s'arrêteront plus aux portes de l'école. La formation permanente, à vie, grâce au temps libéré du travail, sera la règle dans ce monde où nous deviendrons tous, d'après l'expression de Marshall MacLuhan, des «cueilleurs d'information » (MacLuhan 1968).

Dans un article disponible sur Internet (Dyson et al. 1994), le célèbre futurologue Alvin Toffler et trois co-auteurs nous livrent un tableau éloquent de ces

Nébuleuse sémantique, parce que si des expressions sont récurrentes comme décentralisation ou réseau, il n'en reste pas moins que leur définition n'est pas stabilisée. On peut parfois avoir l'impression que le slogan l'emporte sur la conceptualisation, avec tout ce que cela comporte de flou et de confusion, voulus ou non. 
transformations présumées. Utilisant l'expression de Cyberspace ou de Knowledge Age pour qualifier les changements en cours ${ }^{4}$, les auteurs opposent deux séries de termes, la deuxième étant caractéristique de ce qui est en devenir. Ainsi, on découvre les oppositions entre une «limited matter» et un «unlimited knowledge», entre ce qui est centralisé et ce qui est décentralisé, entre la bureaucratie et la distribution du pouvoir, entre ce qui est efficace mais peu accueillant et ce qui est accueillant parce que personnalisé ou encore entre ce qui résiste et ce qui coule, flotte, s'ajuste. Les auteurs estiment que nous passons d'une ère de production, médias et culture de masse à une civilisation démassifiée. Pour eux, «the complexity of Third Wave society is too great for any centrally planned bureaucracy to manage. Demassification, customization, individuality, freedom - these are the keys to success for Third Wave civilization» (Dyson et al. 1994). Bien entendu, d'autres textes nous proposent ce genre de perspectives. Ainsi, Fukuyama estime-t-il que

\begin{abstract}
alors que les principales technologies de la fin du $19^{\mathrm{e}}$ et du début du $20^{\mathrm{e}}$ siècles - pétrole, chimie, automobile, énergie nucléaire... - favorisaient les économies d'échelle et la centralisation, les technologies de la fin du $20^{\mathrm{e}}$ siècle semblent encourager la flexibilité et la décentralisation. L'émergence d'une information extrêmement mobile et bon marché va dans le sens de la démocratisation: les institutions - gouvernements, entreprises, syndicats... - voient désormais leur mainmise sur l'information remise en cause, alors qu'ils s'en servaient autrefois pour manipuler les individus. Ce n'est donc pas un hasard si les régimes autoritaires ont commencé à s'effondrer partout dans le monde au moment même où l'économie mondiale entrait dans l'âge de l'information (1999: 19).
\end{abstract}

\title{
Pour Joël de Rosnay,
}

\begin{abstract}
la puissance informatique personnalisée renforce l'autonomie et l'indépendance des «travailleurs du savoir», nouveaux nœuds des réseaux neuronaux planétaires. L'individu devient la cellule «entrepreneuriale » de base du système productif et créatif global. Une personne associée à sa logistique informatique et communicationnelle peut disposer désormais d'une capacité d'influence et d'action équivalente à celle d'une entreprise centralisée de l'ère taylorienne. En quelques clics de souris, elle peut voyager dans l'espace et dans le temps, économisant énergie et argent, densifiant ses réseaux et répondant rapidement aux demandes de ses clients (1995: 239).
\end{abstract}

Ce qui est intéressant, c'est que l'on trouve dans ces textes aussi bien ce que l'on peut appeler une rhétorique libertaire qu'une rhétorique libérale. D'un côté, les aspirations à vivre dans une société transparente, basée sur de petites communautés, où la liberté de parole serait complète et, de l'autre côté, les tentatives d'imposer les lois du marché dans une grande partie des activités humaines épargnées jusque là trouvent une forme de terrain d'entente. L'idée d' «alliance» proposée par Philippe Breton (1996: 29) met peut-être trop l'accent sur l'aspect antiétatiste des discours. Pour lui, en effet, les discours libéraux et libertaires sont alliés contre les discours mettant en avant le rôle essentiel de l'Etat dans le développement des technologies de l'information et de la communication (TIC). C'est une partie du problème mais il ne faudrait pas oublier que nous avons plutôt affaire à une redéfinition des organisations et des institutions en général.

\footnotetext{
4 Liés à la fameuse «troisième vague » chère à Toffler (voir Toffler 1970).
} 
Il est important de noter maintenant que de nombreux gouvernements et de nombreuses organisations internationales ont produit des discours sur la société de l'information. On peut dire que ces discours ont pris une dimension internationale, non seulement parce qu'ils abordent inévitablement la question de la mondialisation ou de la globalisation, mais surtout parce qu'ils proviennent du monde entier (Brésil, Japon, Union européenne, Etats-Unis mais aussi Afrique ${ }^{5}$ ) et que des organisations comme l'OCDE, l'UNESCO, la CEA ${ }^{6}$ et beaucoup d'autres font de cette question une de leurs priorités. En étudiant de près les divers rapports ou discours issus de ces pays ou organisations, on peut à nouveau repérer des éléments liés à la décentralisation, la flexibilité ou la formation permanente. Ainsi, dans un texte de l'OCDE, on peut lire que,

\begin{abstract}
Today's children need to be prepared for a life in which they will change jobs or job responsibilities frequently and will be regularly retrained as work environments evolve. This preparation must take place in classrooms which bring together students from diverse backgrounds and cultures. In the past, the young received education over a fixed period in preparation for work and life. In the new knowledge-based economy, however, continuous re-education will be the norm, so that skills remain relevant, individuals employable, and firms able to adapt and compete (OCDE 1997: 116).
\end{abstract}

Un autre exemple intéressant nous est proposé par le Sénat français:

\begin{abstract}
Les nouvelles technologies de l'information et de la communication permettant de tisser à une vitesse foudroyante, avec Internet, une toile mondiale qui, déjà, relie plus de 100 millions d'être humains, et qui devrait fondre dans une même communauté plus d'un milliard d'hommes avant 10 ans, posera inexorablement des problèmes fondamentaux à notre société pyramidale héritée de la Rome antique et de la Rome catholique. (...) Il serait dommageable, pour notre pays, que ses gouvernants, hauts fonctionnaires et aussi chefs d'entreprise qui se croient à l'abri, depuis des siècles, en haut de leur structure ne sentent pas que la mutation profonde portée par les réseaux est en train d'imbiber la base de nos vieilles pyramides, et ne pressentent pas la nécessité de ressourcer leur légitimité dans cette nouvelle eau de jouvence (Trégouët 1998: 10-11).
\end{abstract}

Le constat général est, en fait, très proche de ce que l'on trouve dans la littérature «savante».

Est-il nécessaire alors d'insister sur la ressemblance entre les textes consacrés à la société de l'information et la littérature de management étudiée par Boltanski et Chiapello? On retrouve les mêmes éléments fondamentaux dans les deux corpus et la question de savoir si les discours concernant la société de l'information véhiculent, en partie, «le nouvel esprit du capitalisme» devient non seulement légitime mais certainement pertinente. Mais on ne peut en rester là. Le fait que certains richissimes chefs d'entreprises comme Bill Gates (voir Gates 1995) ainsi que des gouvernements et des organisations internationales défendent l'idée d'une société de l'information, et la manière dont cela se fait, en combinant subtilement, par exemple, des discours sur l'inéluctabilité du changement et la néces-

5 Voir, par exemple, le discours de Nelson Mandela à l'ouverture de Telecom 98, en Afrique du Sud. «As the information revolution gathers yet more pace and strikes deeper roots, it is already redefining our understanding of the world. Indeed the speed of technological innovation could bring the ideal of a global village sooner than we thought possible» (Mandela 98).

6 Commission économique pour l'Afrique (ONU). 
sité, pourtant, d'agir pour ne pas prendre de retard sur la concurrence internationale, nous incite toutefois à nous demander s'il ne serait pas pertinent d'introduire dans notre réflexion le concept d'idéologie.

\section{LA «SOCIÉTÉ DE L'INFORMATION» COMME IDÉOLOGIE?}

Toutes sortes d'expressions pour qualifier les discours sur la société de l'information ont été utilisées. Dans un seul et même article, Bernat Lopez (1996) indique, par exemple, que la société de l'information est à la fois un concept, un mythe, une utopie ou un slogan néo-libéral. Ailleurs, on retrouvera, parmi d'autres expressions possibles, l'idée que la société de l'information est une religion ou une fiction. La notion d'idéologie est peut-être une de celles que l'on retrouve le plus souvent. Elle a quelque chose de séduisant en ce sens qu'elle permet de mettre l'accent sur la volonté qu'a un groupe de maintenir l'ordre présent et qu'elle peut laisser entendre qu'il y a distorsion, volontaire ou non, de la réalité en fonction d'intérêts particuliers (voir Gabel 1999). Reste que l'on a affaire à un concept polysémique et qu'il faut donc l'utiliser avec prudence. En effet, certains auteurs utilisent ce qu' on pourrait appeler une version relativement neutre ou neutralisée du concept. On l'a vu plus haut avec la définition proposée par Boltanski et Chiapello. Pour eux, l'idéologie est ancrée dans la réalité et il est hors de question de n'y voir qu'un discours visant à voiler des intérêts matériels. On le voit également dans la définition proposée, par exemple, par Philippe Breton. Pour lui, l'idéologie est

une manière de se représenter globalement la société, ce qu'elle est, mais aussi ce qu'elle devrait être, ou ce qu'elle devrait rester. L'idéologie constitue le prolégomène obligé, au plan de la représentation, de l'action, ou de l'absence d'action - qui en est une forme particulière. L'idéologie se reconnaît facilement à ce qu'elle fournit les raisons du préférable (Breton 1996: 24).

L'idée d'intérêts particuliers et de voilage n'est donc pas vraiment présente. Par contre, l'accent est mis sur l'action. Quand Armand Mattelart (2001, en particulier pp. 3-4), Kees Brants (1989, en particulier, p. 79 et 82) ou Nicholas Garnham utilisent la notion d'idéologie, on est tenté de l'interpréter dans un sens plus critique bien que ne soient pas toujours proposées de réelles définitions. Pour Garnham,

La théorie de la 'société de l'information' a maintenant pris la place de paradigme dominant. L'affronter en tant que théorie scientifique et en tant qu'idéologie est désormais inévitable. Voilà en effet une théorie de la communication qui se présente partout comme un moyen de comprendre la période historique actuelle et les tendances de développement dominantes dans notre société, mais aussi simultanément comme l'idéologie de légitimation privilégiée des détenteurs du pouvoir économique et politique (Garnham 2000: 55).

L'accent est mis ici sur la légitimation et les rapports de domination. Il semble inévitable de devoir se poser ce type de questions. C'est peut-être chez Paul Ricœur que l'on trouve une réflexion sur l'idéologie qui peut relier ces différents 
points de vue. Pour lui, l'idéologie a trois fonctions : celles de distorsion ${ }^{7}$, de légitimation et d'intégration. Elle a donc des fonctions aussi bien positives que négatives, un rôle aussi bien constructif que destructeur (Ricœur 1997: 17). D'un côté, l'idéologie peut avoir un aspect dissimulateur et peut être liée à des problématiques de pouvoir quand elle vise à justifier ou légitimer le système d'autorité tel qu'il est. Par contre, d'un autre côté, on ne peut pas admettre une séparation fondamentale entre l'idéologie et la réalité qui ferait de la première une pure illusion ou un reflet des rapports de force économique. En effet, l'idéologie a une fonction d'intégration sociale fondamentale et peut être considérée comme un moteur de l'action humaine. Pour Ricœur, il est impossible de penser séparément la réalité et l'imaginaire, ceci étant dû à la nature symbolique de la vie sociale. C'est ce qui permet de concevoir l'idéologie comme étant à la fois un moteur de l'action et une distorsion de la réalité. «C'est seulement parce que la structure de la vie sociale des hommes est symbolique qu'elle est susceptible de distorsions. Si elle n'était pas symbolique dès le début, elle ne serait pas distordue. La possibilité de la distorsion est une possibilité ouverte par cette fonction [symbolique]» (Ricœur 1997: 28).

En fin de compte, la notion d'idéologie, malgré les différences de définitions qui existent et parce qu'elle offre la possibilité d'une approche critique de la légitimation, a ceci d'intéressant qu'elle peut nous pousser à interroger de plus près la réalité et à le faire en mettant l'accent sur des questions de pouvoir ou de domination sans pour autant faire de l'idéologie une justification a posteriori et sans rapport avec la réalité. Il s'agit bien, dans ce cas, de se demander si les discours sur la société de l'information n'incitent pas à construire une réalité en occultant les rapports de force qui tendent à maintenir l'ordre dominant, inégalitaire et contraignant.

\section{QUELLE RÉVOLUTION DE L'INFORMATION?}

Dans un article extrêmement intéressant, Frank Webster et Kevin Robins (1989) mettent en cause l'idée d'une transformation fondamentale liée aux développements de l'informatique et des télécommunications. Tout au plus, ces technologies contribuent-elles à renforcer des tendances trouvant leurs origines il y a de cela au moins un siècle. En effet, pour eux, il est nécessaire de rappeler que l'appropriation de l'information a toujours été un aspect constitutif des sociétés capitalistes, sans que le développement des technologies soit déterminant. S'il fallait vraiment parler d'une révolution de l'information et de la naissance d'une société de l'information, ce qui est de toute manière problématique, il faudrait plutôt les considérer du point de vue des transformations dans le domaine du contrôle et de l'accès différencié (et inégal) à l'information. Pour Webster et Robins, un moment particulièrement déterminant dans l'histoire se situe alors au tout début du $20^{\mathrm{e}}$ siècle, au moment où apparaît le Scientific Management de Frederick Winslow Taylor. Pour ce dernier, une production efficace est fondamentalement liée à un contrôle et une surveillance des ouvriers. De plus, tout le travail de conception ou de planification doit être retiré à ces ouvriers et centralisé dans les

7 Qui peut être distorsion de la pensée par les intérêts ou même dissimulation. 
départements ad-hoc. Ceux-ci sont chargés, entre autres, de l'étude détaillée des mouvements des travailleurs ou du temps accordé à chaque tâche, étude aboutissant à une standardisation de ces dernières comme à une standardisation des outils ou des produits. Le taylorisme est en fait devenu bien plus qu'une doctrine de management d'entreprise et on peut même considérer qu'il est devenu une nouvelle philosophie sociale. En-dehors de l'entreprise, le Scientific Management est devenu une nouvelle forme de contrôle social, non seulement dans le sens de la domination ou de la surveillance, mais aussi dans le sens de la capacité qu'a une organisation sociale de se réguler. En effet, Taylor et ses successeurs pensaient que l'idée d'un management et d'une régulation rationnels, scientifiques et efficaces pourrait être étendue au-delà de la place de travail à d'autres activités sociales. Ainsi s'est développée l'idée qu'une société pouvait devenir efficace, harmonieuse, sous la direction d'experts compétents gérant aussi bien la production que la consommation des biens et des idées. Que ce soit dans le domaine politique ou économique, ces principes ont certainement participé au mouvement qui a vu l'émergence des idéaux technocratiques, de la rationalisation de l'administration, du marketing, de la publicité ou encore de la propagande. Tout cela est lié, on le voit, à des formes de contrôle ou de maîtrise de l'information. Le développement des TIC, nouvelles ou pas, serait donc à inscrire dans ce contexte-là et permet d'imaginer un renforcement de logiques anciennes plutôt que la réalisation d'une utopie réticulaire. En fait, pour Webster et Robins, la fonction des TIC a été double en vue d'assurer la cohésion et la reproduction des sociétés capitalistes; d'un côté, les technologies ont permis le développement et le renforcement de mécanismes de management, planification ou administration et, de l'autre côté, les technologies ont été au centre de stratégies de surveillance et de contrôle.

Il devient intéressant de relever que, dans l'analyse de Boltanski et Chiapello, les deux «derniers » esprits du capitalisme restent, malgré l'apparition importante, dans le dernier $\operatorname{cas}^{8}$, de la «cité par projets », dominés par une logique industrielle (Boltanski et Chiapello 1999: 204). Or, dans leur modèle, la «cité industrielle»" et le «monde industriel» qui rend compte de la mise en pratique des principes de la cité ${ }^{10}$ sont basés, en particulier, sur les notions d'efficacité, de performance et de productivité. L'action, dans le monde industriel, est conduite par la méthode scientifique et «les opérations de standardisation, de formalisation, permettent de voir le monde par des données exprimées en nombre, chiffrées, prêtes à être traitées, cumulées, additionnées» (Boltanski et Thévenot 1991: 260). Dans ce monde, les «petits» sont ceux qui sont improductifs parce qu'ils fournissent peu de travail, sont absents ou inactifs (chômeurs ou handicapés, par exemple), démotivés ou trop rigides, inadaptés. C'est «l'homme d'action» qui est mis en avant car «l'absence d'utilisation des potentiels humains disponibles est une grave

8 Celui du «nouvel esprit du capitalisme».

9 Dont Saint-Simon (1760-1825) est le porte-drapeau (Boltanski et Thévenot 1991: 151). Sur les liens entre Saint-Simon, l'industrialisme, les réseaux de communication et la réalisation d'une communion planétaire, on lira avantageusement les travaux de Pierre Musso (nota. 1999: 78-83). Webster et Robins relèvent aussi le lien entre Saint-Simon et les tendances technocratiques visant à une résolution des affaires publiques par des experts plutôt que par le biais des citoyens dans un espace public (1989: 341).

10 Boltanski et Thévenot (1991: 252-262). 
atteinte à la dignité des gens » (Boltanski et Thévenot 1991: 255). Il faut donc être productif, efficace, et la production doit être dirigée par des experts. Une étude en termes de continuité plutôt qu'en termes de changement prend donc une fois de plus tout son sens car ces éléments, semblables à ceux relevés par Webster et Robins, sont en effet au cœur de nombreux discours sur la société de l'information même s'ils ne frappent plus, tant ils sont devenus évidents. En fait, dans les discours sur la société de l'information, le bien-être social est régulièrement subordonné aux nécessités de la productivité économique.

On remarquera que le même type d'analyse est possible avec la logique marchande, d'ailleurs fortement liée à la logique industrielle ${ }^{11}$. Dans les textes analysés par Boltanski et Chiapello comme dans la littérature sur la société de l'information, sa présence n'est de loin pas négligeable (Boltanski et Chiapello 1999: 204; Lopez 1996) ${ }^{12}$. En fait, beaucoup de discours sur la société de l'information ${ }^{13}$ semblent jouer sur deux plans qui les mènent à promouvoir la logique marchande de compétition dans le même temps qu'une logique de projet ou de réseau. Or, dans le «monde marchand», «les spécificités, les attaches personnelles et les liens locaux sont des particularismes dont on doit se libérer pour accéder à un marché anonyme et sans frontières » (Boltanski et Thévenot 1991: 321). On retrouve bien l'écho des appels insistants à la mobilité. Cette mobilité, la flexibilité ou l'adaptabilité sont souvent présentées, d'une part, comme une possibilité, celle de construire sa carrière, et, d'autre part, comme une nécessité car elles permettent d'alimenter continuellement en travailleurs un marché particulièrement fluctuant. L'élément contraignant est donc très fort. Les dispositifs qui pourraient assurer le maintien d'une justice dans un monde connexionniste ne semblent pas non plus toujours le principal souci des défenseurs de la société de l'information. La logique concurrentielle l'emporte facilement sur l'épanouissement des individus et, dans les discours internationaux, la compétition l'emporte largement sur la coopération malgré les tentatives visant à montrer que ces deux manières de comprendre la mondialisation ne sont pas contradictoires. On peut noter enfin qu'une société rendue transparente par les «nouvelles» technologies en réseau peut être vue comme un moyen idéal de rendre possible le marché global.

Dans le cadre même des discours, on peut donc estimer que le modèle de la décentralisation ou la «cité par projets» occultent souvent, par leur aspect clinquant et prétendument novateur, d'autres éléments fondamentaux d'une définition du monde tel qu'il est et tel qu'il devrait être. La logique industrielle et la logique marchande sont présentes dans les discours mais leur importance est, délibérément ou non, masquée par l'accent mis sur la révolution informationnelle ou le

11 L'expression de «capitalisme industriel» ayant ici toute sa pertinence pour qualifier le système social dans lequel nous vivons.

12 Même si, on l'a dit plus haut, la logique marchande n'est pas omniprésente.

13 En particulier en provenance des gouvernements ou organisations internationales; voir, notamment, les productions du G8. Dans une charte récente, on lit ainsi que l'une des bases pour «faciliter le passage à une société de l'information» serait «des réformes économiques et structurelles qui favorisent un environnement caractérisé par l'ouverture, l'efficacité, la concurrence et l'innovation, avec l'appui de politiques axées sur des marchés du travail adaptables, sur le développement des ressources humaines et sur la cohésion sociale» (G8 2000). Cela se retrouve aussi très clairement, par exemple, chez Gates (1995) et Dyson (1994). 
développement des réseaux. Il y a donc là une dimension idéologique. On peut en découvrir un autre aspect en étudiant d'un peu plus près les pratiques effectives. En effet, si tous les discours sur la société de l'information n'ont pas vocation idéologique, ils en sont malgré tout les complices, quand, fonctionnant sur le modèle de l'utopie post-industrielle ou libertaire, ils proposent une représentation du monde qui ne tient justement pas compte des forces dominantes dans notre société ou des problèmes engendrés par l'accent mis sur la mobilité totale.

\section{UNE SOCIÉTÉ DE L'INFORMATION INQUIÉTANTE}

En fait, trois problèmes fondamentaux et fortement imbriqués entre eux semblent particulièrement négligés. Premièrement, on peut penser à la question des inégalités. On n'insistera pas sur cette question mais il est patent que le développement des TIC ne concerne pas tous les pays ou toutes les couches de la population de la même manière. Il est encore loin le jour où les habitants des pays du Tiers-Monde profiteront tous d'une connexion Internet ou d'un abonnement à un téléphone mobile. Et si cela devait arriver un jour, faudrait-il encore que l'usage de ces technologies permette réellement le développement de la démocratie universelle ou du marché global égalitaire qui nous sont parfois promis. On peut en douter. Le contexte du développement des technologies est fortement concurrentiel et l'ouverture des marchés préconisée par certains pays n'a pas forcément pour but une égalisation des forces ou une amélioration de l'accès à une éducation de qualité. On se rappellera, par exemple, des tentatives, au sein de l'OCDE, de faire signer aux pays de ce monde l'AMI (Accord Multilatéral sur l'Investissement $)^{14}$ dont les objectifs étaient clairement d'imposer la présence et les règles d'entreprises transnationales à des pays qui se seraient vus obligés d'accueillir, à quasi n'importe quelle condition et sans restrictions, ces entreprises étrangères. Le développement des TIC ne peut être extrait de ce contexte-là, tant il est vrai qu'elles favorisent l'accroissement des activités des entreprises à une échelle mondiale. Il faut aussi considérer les problèmes liés à la culture, aux médias et à la formation en général. Il y a de formidables potentiels d'imposition de normes sociales par les grandes puissances maîtrisant les réseaux d'information ou de communication. Il n'est pas sûr que la possibilité qu'aurait chaque individu de produire de manière autonome de l'information résiste aux grandes entreprises d'informatique, télécommunications, télévision et autres producteurs géants d'information. Herbert Schiller (1996) a, par exemple, clairement dénoncé, aux EtatsUnis, la mauvaise qualité de l'information transmise par les différents médias (divertissements de petit niveau, censure, contrôle du contenu par les grands groupes) ou la privatisation de l'éducation (publicité à l'école, dégradation de l'enseignement pour certaines catégories de la population). Il est évident que l'égalité devant l'information, le savoir et l'éducation n'est de loin pas acquise à l'intérieur même de chaque nation, serait-ce la plus «avancée ».

Deuxièmement, on ne peut éviter de s'interroger sur les problèmes liés au contrôle, au sens large, et à la surveillance des travailleurs et de la population en

14 Voir Wallach (1998). 
général. Il ne s'agit pas de se laisser tenter par une vision trop pessimiste, qui suspecterait un pouvoir occulte de surveiller continuellement tous nos gestes. Toutefois, on ne peut pas non plus céder à une utopie de la transparence sans se demander alors où se situe la liberté de chacun et sa vie privée. On se rappellera quand même que l'idée de web comporte une double signification intéressante. La toile permet de se déplacer partout et rapidement (c'est l'idéal d'Internet, du world wide web) mais elle est aussi l'instrument qui permet à l'araignée d'attraper sa proie. La question du contrôle va bien sûr plus loin que ces métaphores. Ainsi, on peut craindre le développement de la vidéosurveillance dans les villes ${ }^{15}$ ou certaines formes d'espionnage économique comme semblent l'avoir pratiqué notamment les Etats-Unis et la Grande-Bretagne grâce au système Echelon ${ }^{16}$. La question du contrôle permet aussi de prolonger les analyses de Webster et Robins. On peut penser notamment aux outils du knowledge management qui devraient permettre de «capturer la connaissance ${ }^{17}$ dans un monde vu comme complexe et fluctuant. Les «savoir-faire» des employés devenant eux aussi de plus en plus complexes et les employés de plus en plus mobiles, il s'agit de trouver un moyen de récupérer et garder ces compétences ou connaissances pour que les entreprises ne dépendent pas d'une personne particulière. Les principes tayloriens sont toujours en vigueur mais pour de nouvelles catégories de métier. Le développement extraordinaire des normes ISO (International Organization for Standardization) peut être interprété dans le même sens. De plus en plus d'activités sont normalisées et le contrôle de ces normes devient bien entendu un enjeu fondamental, pour le meilleur et pour le pire.

Enfin, troisièmement, il ne faudrait pas négliger l'impact que peuvent avoir les nouveaux modèles normatifs véhiculés par les discours sur la société de l'information. On peut sérieusement craindre une forte déstabilisation des individus confrontés à des incitations continuelles à la mobilité, à la flexibilité, au changement permanent ${ }^{18}$ ou à la formation continue. Les travaux d'Alain Ehrenberg qui mettent en parallèle les nouveaux modèles du sport (la compétition), des vacances (la consommation ou l'épanouissement personnel), de l'entreprise (la concurrence) ainsi que les nouvelles représentations de la souffrance psychique (la dépression comme une pathologie de l'action) nous montrent bien les dangers de cette vision du monde. Un modèle de l'humain, basé sur «la valorisation de l'individu souple, mobile, autonome, indépendant, qui trouve par lui-même ses

15 Philippe Breton et Luc Gwiazdzinski s’inquiètent par exemple du développement de la technologie sécuritaire à Strasbourg. La multiplication des installations de vidéosurveillance contribue au risque de rendre désert l'espace public. «Ceux qui regardent, horrifiés et critiques, les 'villes privées' qui naissent Outre-Atlantique, où les classes privilégiées ont choisi un développement séparé d'avec les classes défavorisées, doivent savoir que c'est bien la vidéosurveillance, et demain ses prolongements sur Internet, qui est l'outil de ce véritable apartheid social» («Strasbourg, capitale de la technologie sécuritaire», Le Monde du 28 janvier 2000).

16 Voir Rivière (1999).

17 Expression empruntée à un cours consacré aux systèmes d'information documentaire et donné à des étudiants post-grades de l'Université de Genève, en décembre 2001.

18 On notera, par exemple, le conseil donné par l'IMD (International Institute for Management Development de Lausanne) à ses clients; «appliquez le changement, même s'il n'y a aucune raison apparente de le faire». "C'est une question de dynamique » nous révèle un des professeurs de l'institution (Le Temps du 14 décembre 2001, cahier «Emploi et formation », p. 3). 
repères dans l'existence et se réalise par son action personnelle» (1996: 15), est en train de s'imposer avec tous les problèmes de représentation de soi et de constitution de l'identité que cela comporte lorsque toute base solide est constamment remise en cause. Les individus doivent être à même de s'auto-motiver, «être les entrepreneurs de [leurs] propres vies » (1996: 16), ce qui peut d'ailleurs se révéler être un puissant outil d'auto-contrôle. Il n'est plus besoin de surveiller les individus pour qu'ils travaillent. Ils s'en chargent eux-mêmes. De plus, le management s'intéresse maintenant au «savoir-être » des individus, ce qui permet d'instrumentaliser «les propriétés les plus humaines des êtres humains, leurs affects, leur sens moral, leur honneur, leur capacité d'invention» (Boltanski et Chiapello 1999: 152). L'humain est toujours, d'une certaine manière, considéré comme une machine ou, autre manière de voir les choses, comme un «homme-marché » ${ }^{19}$.

On aura donc vu que, si l'on peut admettre que les discours sur la société de l'information véhiculent en partie le «nouvel esprit du capitalisme», ils le font aussi en occultant certains faits liés aux logiques industrielles et marchandes qui régissent actuellement nos sociétés. D'autre part, ils négligent les conséquences négatives du développement d'un nouveau modèle de l'existence sociale basé sur les notion de réseau, décentralisation ou mobilité. Une approche en terme d'idéologie a certainement pour avantage d'interroger les discours sur leurs fonctions et leur rapport à la réalité et permet donc de contester ce que nous avance toute une littérature laudative ou complaisante sur la société de l'information. En se basant sur un nouveau modèle du bien commun, bien analysé par Boltanski et Chiapello dans «Le nouvel esprit du capitalisme», ces discours nous invitent en effet à détourner le regard de phénomènes sociaux plus stables ou ancrés dans l'histoire. Ils ont donc un rôle idéologique que l'on ne peut pas négliger, d'autant plus qu'ils contribuent certainement à construire la réalité sociale en accompagnant, en particulier, le développement des TIC. Comme l'indiquent Webster et Robins,

\begin{abstract}
among the significant issues to be raised by the new information technologies are their relation to social forms of organization, their centrality to structures of political power, and their role in the cultural logic of consumer capitalism. Sociological analysis is naïve, we believe, when it treats the new telecommunications, space, video, and computing technologies as innocent technical conceptions and looks hopefully to a coming, postindustrial Utopia. Better to look back to the past, to the entwined histories of reason, knowledge, and technology, and to their relation to the economic development of capitalism and the political and administrative system of the modern nation state (1989: 346).
\end{abstract}

Nous ne pouvons qu'essayer de répondre à cet appel.

Institut d' anthropologie et de sociologie

Université de Lausanne

19 «Un individu d'un type nouveau, en étroite symbiose avec les rythmes marchands, recyclable à volonté, flexibilisé à outrance, jetable tel un mouchoir en papier usagé, cloné indéfiniment selon les lignes de force de la philosophie managériale branchée: l'homme 'programmé' par et pour le marché, l'homme-marché » (Arondel 1997: 11). 


\section{BIBLIOGRAPHIE}

Arondel, Philippe

1997 L'homme-marché. Paris: Desclée de Brouwer.

Boltanski, Luc et Ève Chiapello

1999 Le nouvel esprit du capitalisme. Paris: Gallimard.

Boltanski, Luc et Laurent Thévenot

1991 De la justification. Les économies de la grandeur. Paris: Gallimard.

Brants, Kees

1989 «The social construction of the information revolution». European journal of communication vol. 4 : 79-97.

Breton, Philippe

1996 «Faut-il appliquer le principe de laïcité à Internet? Bref plaidoyer pour une séparation de l'idéologique et du technique ». Terminal 71-72: 23-31.

Duff, Alistair

2000 Information society studies. London: Routledge.

Dyson, Esther et al.

1994 Cyberspace and the American Dream: A Magna Carta for the Knowledge Age. http://www.pff.org/position old.html: [17.12.2001, sur le site de] The Progress and Freedom Foundation.

Ehrenberg, Alain

1996 Le culte de la performance. Paris: Hachette (première publication 1991).

Fukuyama, Francis

1999 «La post-humanité est pour demain». Le Monde des débats 5: 16-20.

G8

2000 La charte d'Okinawa sur la société mondiale de l'information. http://www.g8.gc.ca/2000/Okinawa_Charter_GIS0722-f.asp: [17.12.2001, sur le site de] Gouvernement du Canada.

Gabel, Joseph

1999 «Idéologie». Dans Encyclopaedia Universalis (CD-rom). Paris.

Garnham, Nicholas

2000 «La théorie de la société de l'information en tant qu'idéologie. Une critique». Réseaux 101: 55-91.

Gates, Bill

1995 La route du futur. Paris: Robert Laffont.

Lopez, Bernat

1996 «a ‘société de l'information'. Promesse du futur ou slogan néo-libéral?». Médiaspouvoir 43-44: 103-113.

MacLuhan, Marshall

1968 Pour comprendre les médias. Paris: Mame/Seuil (première publication en anglais 1964).

Mandela, Nelson

1998 Speech by President Nelson Mandela at the opening of Africa Telecom98. http://www.itu.int/TELECOM/aft98/overview.html: [18.02.2002, sur le site de] International Telecommunication Union (ITU).

Mattelart, Armand

2001 Histoire de la société de l'information. Paris: La Découverte.

Musso, Pierre

1999 «La symbolique du réseau». Quaderni 38: 69-98.

OCDE

1997 Information technology outlook. Paris: OCDE.

Ricœur, Paul

1997 L'idéologie et l'utopie. Paris: Seuil (première publication en anglais 1986). 
Rivière, Philippe

1999 «Le système Echelon». Manière de voir 46: 40-42.

Rosnay, Joël de

1995 L'homme symbiotique. Regards sur le troisième millénaire. Paris: Seuil.

Schiller, Herbert

1996 Information inequality, the deepening social crisis in America. New York: Routledge.

Simioni, Olivier

2000 «La société de l'information: émergence et multiplication des discours». Dans La «société de l'information»: une idée confuse?, G. Berthoud et al. Lausanne: Institut d'anthropologie et de sociologie, pp. 13-39.

Toffler, Alvin

1980 La troisième vague. Paris: Denoël.

Trégouët, René

1998 Des pyramides du pouvoir aux réseaux de savoirs. Comment les nouvelles technologies de l'information vont aider la France à entrer dans le $21^{e}$ siècle. Paris : Les rapports au sénat.

Wallach, Lori

1998 «Le nouveau manifeste du capitalisme mondial». Le Monde diplomatique février: 22.

Weber, Max

1964 L'éthique protestante et l'esprit du capitalisme. Paris: Plon (première publication en allemand 1905).

Webster, Frank et Kevin Robins

1989 «Plan and control. Towards a cultural history of the information society». Theory and society $18: 323-351$. 